\section{P77 (continued)}

Background (Background, Rationale, Prior Research, and/or Theory): Programs to help individuals with cancer and their caregivers address nutrition-related behaviors must reflect the needs, interests, and preferences of these unique populations.

Objective: To characterize nutrition-related beliefs, experiences, and behaviors among individuals with cancer and their caregivers, a critical step in development of effective nutrition interventions; and to identify preferences, barriers, and facilitators of engagement in lifestyle modification strategies.

Study Design, Setting, Participants, Intervention: A convergent mixed methods study design was used. Individuals with cancer and their caregivers were recruited from American Cancer Society's Philadelphia Hope Lodge to complete surveys and in-depth interviews. NCI's Food Attitudes and Behaviors Survey was used to assess foodrelated attitudes, preferences, beliefs and behaviors.

Outcome Measures and Analysis: Descriptive statistics were calculated. Interviews were recorded, transcribed verbatim, and analyzed using inductive thematic analysis. Results: Thirty-nine individuals with cancer $(n=19,67 \%$ male, $73 \%>55$ yrs) and their caregivers $(n=20,31 \%$ male, $63 \%>55$ yrs) completed study activities. Three themes emerged: challenges managing treatment side effects, uncertainty regarding "healthy" foods, and desire for nutrition support for healthy survivorship. Caregivers reported lower nutrition-related self-efficacy compared to patients. Fewer than $20 \%$ of caregivers and 33\% of patients felt they understood government nutrition recommendations. Sixtyseven percent of patients and $75 \%$ of caregivers reported they believed what they eat affects their health, yet there was disagreement among participants regarding what comprises "poor" vs. "good" nutrition. Barriers to eating fruits and vegetables among patients included lack of satiation, availability, and preparation time; and among caregivers included spoilage and availability. Participants reported interest levels in nutrition and cooking classes and therapeutic gardening.

Conclusions and Implications: Nutrition intervention programs should be tailored for cancer survivors and caregivers who are experiencing risks associated with management of treatment side effects, need to improve their understanding of healthy foods to eating during recovery, and for those who are more comfortable with a program that can address specific concerns related to the cancer experience.

Funding: Pennsylvania Department of Health.

\section{P78 Outcome Evaluation of Alabama} Supplemental Nutrition Assistance ProgramEducation (SNAP-Ed) Social Marketing Campaign

Brent Walker, MS, RD, brent.walker@altarum.org, Altarum, 4 Milk Street, Suite 303, Portland, ME 04101; Brenda Wolford, MS, RD; Rebecca Hofer, MPH;

Sondra Parmer, PhD, Alabama Cooperative Extension System, Auburn University; Katie Funderburk, MS, RD; Barb Struempler, PhD
Background (Background, Rationale, Prior Research, and/or Theory): USDA's Food and Nutrition Service (FNS) encourages social marketing as a way to enhance nutrition education in SNAP-Ed. In 2016, the Alabama Cooperative Extension System at Auburn University (ACES) developed three new billboard messages as part of its rebranded Nutrition Education Program, Live Well Alabama.

Objective: To measure awareness of the social marketing campaign messages among selected Alabama SNAPEd parents.

Study Design, Setting, Participants, Intervention: Altarum conducted an outcome evaluation using measures from the SNAP-Ed Evaluation Framework to assess reach and receptivity of social marketing campaign messages among parents of third grade Body Quest students throughout Alabama. A telephone survey was designed and data collected in April and May 2017.

Outcome Measures and Analysis: Descriptive statistics were produced to describe the sample population and outcome variables. Confidence intervals were calculated to estimate significant differences among survey respondents at the alpha $=0.05$ level.

Results: A total of 433 surveys were completed. Over onethird $(37.7 \%)$ of respondents were exposed to at least one of the campaign messages. Repeated exposure to campaign messages was evident, most notably for the fruit and vegetable message where nearly half (46.9\%) of all respondents exposed to this message recalled seeing it five or more times. Respondents exposed to the campaign messages consumed significantly more cups of fruits and vegetables per day than those who were unexposed (1.8 and 1.3 cups, respectively for fruits; 1.9 and 1.6 cups, respectively for vegetables). Respondents most frequently reported planning healthy meals $(46.1 \%)$, eating more fruits and vegetables (44.9\%), buying more fruits and vegetables (41.3\%), thinking about making healthy food choices (40.7\%), drinking more water (38.9\%), and exercising more (34.1\%) after exposure to campaign messages. Lack of time was reported as the primary barrier for adopting behaviors such as healthier eating and physical activity.

Conclusions and Implications: ACES successfully launched a newly rebranded social marketing campaign in 2017 recognized by over one-third of respondents surveyed.

Funding: Supplemental Nutrition Assistance Program-Education.

\section{P79 Population Trends in Unhealthful} Beverages and Foods by Race/Ethnicity Among Low-Income Mothers in California, 2015 to 2017

Fred Molitor,PhD, fred.molitor@csus.edu, California State University Sacramento, 6000 J Street, Sacramento, CA 95819; Celeste Doerr, PhD, Public Health Institute; Lauren Whetstone, PhD, California Department of Public Health; John Pugliese, PhD; Samantha Trammell, BS, California Department of Social Services

Continued on page $S 45$ 